PALABRAS CLAVE

Petróleo

Perforación de pozos

Deforestación

Bosques tropicales

Costos

Aspectos económicos Aspectos ambientales Estimación de proyectos Ecuador

Diego Azqueta

Catedrático de Fundamentos del Análisis Económico,

Departamento de Fundamentos de Economía

e Historia Económica,

Universidad de Alcalá,

Madrid

$\propto$ diego.azqueta@uah.es

Gonzalo Delacámara

Profesor de Fundamentos del

Análisis Económico

Departamento de Fundamentos

de Economía

e Historia Económica,

Universidad de Alcalá,

Madrid

øonzalo.delacamara@uah.es
REVISTA DE LA CEPAL 94・ABRIL 2008

\section{El costo ecológico de la extracción de petróleo: una simulación}

\author{
Diego Azqueta y Gonzalo Delacámara
}

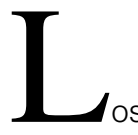

os campos petrolíferos de Ecuador se acercan al término de su vida económica y ya se están otorgando licencias para explotar nuevos yacimientos en la región amazónica. El hecho de que en algunas zonas de mayor valor ecológico del país pueda producirse deforestación, como sucedió en el pasado debido a las migraciones inducidas, cabe preguntarse si no sería razonable explotar estos nuevos yacimientos sin provocar deforestación. Este artículo no da una respuesta categórica pero, a partir de un proyecto de investigación anterior, presenta un ejercicio de simulación en el cual, para mostrar la pérdida económica que acarrearía la deforestación, introduce el valor económico de cuatro servicios prestados por los bosques tropicales. Sostiene además que la evaluación de las consecuencias ambientales debería tener en cuenta la prima correspondiente y se recomienda utilizar un factor de descuento hiperbólico. 


\section{Introducción}

Con el tiempo, la escasez de divisas que enfrentan algunos países en desarrollo se ha traducido en altos niveles de endeudamiento y en una crisis de deuda externa. El peso de esta deuda les plantea serios problemas y dificulta su avance hacia el desarrollo socioeconómico. Ante la necesidad de cumplir con los pagos de la deuda, es posible que los países en desarrollo que tengan problemas de balanza de pagos traten de obtener los recursos necesarios en el mercado financiero internacional. Sin embargo, esto significará que habrán de pagar una alta prima de riesgo por los créditos que obtengan y, en consecuencia, altas tasas de interés. En este caso, la rentabilidad del dólar en dichos países será igual a la tasa de interés y esta será también la tasa interna de rentabilidad social del último proyecto de inversión pública rechazado. Por lo tanto, no sería extraño que al verse obligados a pagar una suma cada vez más alta en concepto de servicio de la deuda, estos países opten por explotar las ventajas comparativas que les otorga su dotación de recursos ambientales, aumentando sus exportaciones de recursos naturales y soportando niveles más bajos de calidad ambiental (Azqueta, 2007). Como se indicará más adelante, el elevadísimo precio de cuenta de la divisa en estos países hace que a corto plazo esta opción parezca económicamente racional. ${ }^{1}$

Sin embargo, lo más probable es que esta búsqueda de nuevas fuentes de divisas no solo resulte insostenible sino que menoscabe, a menudo en forma irreversible, la dotación de recursos naturales. Cálculos económicos de corto plazo podrían respaldar estas prácticas: los permisos de explotación forestal producen la muy necesaria divisa fuerte, mientras que conservar los bosques primarios casi no deja beneficio alguno. Con todo, un cálculo económico elemental puede revelar que tal vez esta no solo sea una asignación menos que óptima de los recursos desde el punto de vista del bienestar general, sino que incluso en términos de racionalidad económica la ganancia neta no sea tan grande y valga la pena buscar mejores formas —aunque quizá más costosas a corto plazo- de explotar los recursos naturales.

Ecuador es un buen ejemplo de esta situación. No solo es un país en desarrollo que tiene graves problemas de pobreza y privación, sino que está muy endeudado con el resto del mundo (cuadro 1). Aunque es probable que ello no se deba exclusivamente a la carga de la deuda, cabe señalar que mientras que en 1990 el gasto público en educación ascendió al 3,1\% del producto interno bruto (PIB) y el gasto público en salud a un $1,6 \%$, en el 2001 y el 2000 estos porcentajes cayeron al $1,1 \%$ y al $0,9 \%$, respectivamente (en los años siguientes mejoraron un poco). Además, mientras que en 1990 se hallaba en situación de pobreza el $62,1 \%$ de la población y de indigencia el 26,2\%, en 1999 las cifras respectivas crecieron hasta el $63,5 \%$ y el $31,3 \%$ (CEPAL, 2007).

CUADRO 1

Ecuador: algunas cifras macroeconómicas

(En dólares de 2000)

\begin{tabular}{|c|c|c|c|c|c|c|c|}
\hline & 1995 & 2000 & 2001 & 2002 & 2003 & 2004 & 2005 \\
\hline Producto interno bruto (PIB) per cápita & 1333 & 1295 & $\ldots$ & 1382 & 1411 & 1501 & 1550 \\
\hline Total deuda externa (en millones de dólares) & 13934 & 13216 & & 16236 & 16756 & 17210 & 17237 \\
\hline Total deuda/PIB (en porcentaje) & 69,0 & 85,1 & & 67,0 & 61,0 & 56,2 & 47,2 \\
\hline Exportaciones de bienes y servicios (en millones de dólares) & 5278 & 5905 & & 5809 & 6366 & 7370 & 7917 \\
\hline Importaciones de bienes y servicios (en millones de dólares) & 5454 & 4939 & & 7194 & 6915 & 7683 & 8720 \\
\hline
\end{tabular}

Fuente: CEPAL, 2007.

\footnotetext{
${ }^{1}$ El precio de cuenta de la divisa indica su escasez real y se expresa como un cociente que muestra la desviación entre este precio sombra (de eficiencia) y el tipo de cambio oficial. En Colombia, durante los
}

años 1980 esta relación fluctuó entre 1,18 y 1,75; en Panamá entre 1,13 y 1,21, y en Venezuela en 1989 fue de 1,08 (Londero, 1992). Lamentablemente, carecemos de esta información para Ecuador. 
Por otra parte, las principales fuentes de divisas - sin contar las remesas de los trabajadores ecuatorianos en el exterior ${ }^{2}$ ponen de manifiesto hasta qué punto el país depende de la explotación de sus recursos naturales: petróleo crudo, bananos y pescado. Con este modelo de especialización, en 2004 el 79\% de las exportaciones ecuatorianas correspondían a productos primarios (un $51 \%$ a energía) y un $14,2 \%$ a productos industriales basados en recursos naturales (CEPAL, 2006).

El modelo de especialización ha traído consigo consecuencias ambientales muy graves:

i) La transformación de los manglares en cultivos de camarones en la costa del Pacífico ha acarreado la desaparición casi total de uno de los ecosistemas mundiales más valiosos desde el punto de vista ecológico (Costanza, D’ Arge y otros, 1997). ii) El uso intensivo de pesticidas, fungicidas y fertilizantes en las plantaciones de bananos se traduce en eutrofización y degradación de los ecosistemas acuáticos. ${ }^{3}$

iii) El aumento acelerado del turismo en las islas Galápagos está produciendo presiones crecientes sobre un entorno muy frágil. En 1997, de los 57 lugares visitados 21 excedían su capacidad de absorción de visitantes. Y algo que podría volverse contra la propia industria turística: a juicio de más del $50 \%$ de los turistas entrevistados, en las islas había congestión, lo que para el 21,8\% de ellos constituía una gran molestia (García, 2000).

iv) Finalmente, la producción de petróleo para la exportación en la región del Amazonas es una nueva amenaza para ese ecosistema único.

Este último es el problema en el que nos gustaría centrar la atención.

\section{II}

\section{Las exportaciones de petróleo de Ecuador}

Ecuador es uno de los mayores exportadores de petróleo de América Latina, pese a que recientemente se ha enfrentado a crecientes dificultades para aumentar su producción. Según la Agencia Internacional de la Energía (Oil Market Report, varios años), en 2002 la empresa estatal Petroecuador, que representa más del $55 \%$ de la producción total de crudo, obtuvo 0,4 millones de barriles diarios, su nivel más bajo en 10 años. Con posterioridad la cifra ha mejorado levemente y en 2006 produjo 0,53 millones de barriles al día.

La mayor parte de su actual producción proviene de la región oriental del Amazonas, conocida como "el Oriente" (yacimientos de Sushufindi, Sacha, Libertador, Cononaco, Cuyabeno, Lago Agrio y Auca), y la mayoría de sus reservas comprobadas, que ascienden a 4.600 millones de barriles, también se encuentran en la parte este de esa región. Por el momento, el petróleo se traslada desde la ciudad de Lago Agrio, en la cuenca amazónica, al terminal de Balao, cerca del puerto de Esmeraldas (costa del Pacífico), a través del Sistema del Oleoducto Transecuatoriano (SOTE). El oleoducto, construido a comienzos del decenio de 1970, ha sido objeto de varias

\footnotetext{
${ }^{2}$ En 2001, las remesas de trabajadores ecuatorianos desde los Estados Unidos ascendieron a 1.400 millones de dólares (Jacôme, 2004).
}

mejoras — la última de ellas en junio de 2000 — y su capacidad es de 300.000 barriles diarios.

Las exportaciones de petróleo probablemente han sido positivas para la economía ecuatoriana. De acuerdo con lo expuesto antes, las divisas generadas por tales exportaciones reducen la necesidad de recurrir a los mercados financieros internacionales y en consecuencia disminuyen la tasa interna de rentabilidad de las inversiones públicas consideradas aceptables, promoviendo potencialmente de ese modo el desarrollo socioeconómico. Sin embargo, esto no debe hacernos olvidar que junto a la atracción de divisas, la extracción de petróleo también está produciendo graves efectos adversos en el medio ambiente. Se sabe por experiencia que las actividades relacionadas con el petróleo (exploración, explotación y traslado del crudo) sin duda tienen sombrías consecuencias ambientales en el ecosistema en que se realizan: en este caso un bosque tropical lluvioso primario (Gupta y Asher, 1998). Tales actividades modificarán los usos de la tierra de manera directa (parte del espacio será ocupado por pozos, caminos, campamentos y otros) e indirecta (habrá nuevas actividades en la zona y probablemente se

\footnotetext{
${ }^{3}$ También está afectando mucho al cultivo de camarones (Colburn, 1997).
} 
abandonarán otras anteriores). Huelga decir que también afectarán al funcionamiento del ecosistema y la corriente de servicios que este puede proporcionar.

Los efectos de las actividades petrolíferas pueden clasificarse en las tres categorías principales que se enumeran a continuación. ${ }^{4}$

i) Efectos directos. Se vinculan directamente con las principales actividades relacionadas con el petróleo (exploración, extracción, traslado y bombeo). Son los siguientes:

- Emisión de contaminantes atmosféricos, por liberación accidental y como consecuencia de la combustión de gas. También habría que incluir los compuestos orgánicos volátiles acumulados en los estanques para residuos del petróleo.

- Vertidos accidentales y reiterados y filtraciones en el suelo y en los sistemas hídricos (aguas subterráneas y superficiales) que podrían causar daños a la salud humana. Un estudio sobre la contaminación de los ríos realizado en el mu- nicipio de Shushufindi en 2001 reveló que la mayor parte de las aguas superficiales tenía un elevado nivel de contaminación química generada por la industria petrolera local, problema que también se detectó en los pozos urbanos. ${ }^{5}$

- Vertidos de agua contaminada al medio ambiente (el agua que trae el petróleo al extraerlo).

ii) Efectos indirectos. No se relacionan directamente con las actividades petrolíferas, pero están muy vinculados a la existencia de tales actividades. Entre ellos cabe mencionar la construcción de nuevos caminos de acceso, que de hecho favorecen el movimiento de potenciales colonos y la colonización de nuevos terrenos.

iii) Efectos inducidos. Son aquellos ligados a actividades secundarias que derivan de las petrolíferas, como el desarrollo de asentamientos urbanos que proporcionan a la población local (trabajadores del sector petrolífero, colonos y otros) servicios básicos de salud, educación, comercio minorista y otros.

\section{III}

\section{Colonización y deforestación}

En la actualidad, los principales campos petrolíferos en explotación se encuentran cerca de la reserva de Cuyabeno, que es una de las áreas ecológicas más valiosas del país. En ella viven seis nacionalidades indígenas diferentes, agrupadas en varias comunidades, a saber: siena, secoya, cofán, quechua (naporunas), shuar y achuar. ${ }^{6}$ Las tres primeras se encontraban allí en la época de la colonización española, mientras que los shuar y achuar llegaron al territorio en calidad de

\footnotetext{
${ }^{4}$ A esto habría que agregar las relacionadas con el trasvase del petróleo, es decir, con el oleoducto. Según un informe independiente emitido en septiembre de 2002 por Robert Goodland, ex jefe del Departamento de Medio Ambiente del Banco Mundial, el nuevo oleoducto en construcción (OCP), que conectará los yacimientos nuevos con la costa, "en gran medida no aplican las políticas de protección social y ambiental recomendadas por el Grupo del Banco Mundial" (Goodland, 2002). Los ambientalistas temen que la construcción provoque graves daños a uno de los santuarios de aves más importantes del mundo: la reserva de bosques tropicales de la selva nublada de Mindo Nambillo, que al mismo tiempo es uno de los destinos importantes del ecoturismo. Tras los daños ocasionados por la construcción de carreteras, en marzo del 2002 el Ministerio del Ambiente de Ecuador suspendió transitoriamente la licencia del OCP. En cuanto al oleoducto existente (SOTE), en junio del 2002 los habitantes de la provincia de Napo ocuparon
}

mano de obra barata en la segunda mitad del siglo XIX y comienzos del siglo XX, con la bonanza del caucho. Los últimos en llegar a colonizar la tierra fueron los naporunas (quechuas), a mediados del siglo XX.

La situación tuvo un vuelco cuando en 1967 la Texaco abrió el primer campo petrolífero de la región (Lago Agrio) y se inició la construcción de caminos. En 1971 se inauguró la carretera que une Quito con Lago Agrio construida por la Texaco, se terminó el camino

\footnotetext{
la estación de bombeo de El Salado y cerraron algunas válvulas, deteniendo el flujo por unos días. En noviembre del mismo año, Petroecuador anunció que desviaría la sección Papallacta-Lago Agrio por el peligro de aludes después de la erupción del volcán Reventador.

${ }^{5}$ En 1993, el pueblo cofán demandó a la Texaco por el presunto vertido de más de 30 mil millones de galones de productos tóxicos en el período 1964-1992. Los tribunales de Nueva York rechazaron la demanda en 1996, sosteniendo que debería haberse presentado en los tribunales ecuatorianos. Sin embargo, en 1998 la Corte de Apelaciones del Estado de Nueva York estimó que el pueblo cofán podía querellarse contra la Texaco en los tribunales estadounidenses, pero la demanda fue rechazada nuevamente en mayo de 2002 por el juez Jed Rakoff del Tribunal Federal del Distrito Sur de Nueva York.

${ }^{6} \mathrm{Al}$ parecer, otro pueblo originalmente del área, los tetetes, se ha extinguido. Lo curioso es que la Texaco dio este nombre al primer pozo de petróleo que entró en funcionamiento.
} 
Lagro Agrio-Tarapoa (construido por PetroecuadorCiudad) y en 1979 se completaron las carreteras Pacayacu-Los Tetetes y Tarapoa-Palma Roja (también construidas por Petroecuador-Ciudad). En menos de 20 años se construyeron en total más de 200 kilómetros de caminos nuevos.

La apertura de estas vías facilitó mucho la llegada de migrantes de otros lugares del país que se instalaron a su vera como agricultores y que quemaron y deforestaron el bosque tropical para convertirlo en tierras cultivables y pastizales. Como resultado, la población de la región oriental aumentó apreciablemente, de 70.000 personas en 1950 a 372.533 en 1990, lo que equivale a un incremento total del 432\%, o anual del 10,8\% (Brown, Digiacinto y otros, 1996). En pocos años llegaron a la zona más de 200.000 colonos. Por lo general, los campesinos ocupaban una franja de 250 metros de longitud y 2.000 metros de ancho paralela a la carretera, a la que agregaban en calidad de reserva otros 2.000 metros, contiguos a los anteriores. Cuando al cabo de cinco o seis años se agotaba la primera porción de terreno despejada, ocupaban la reserva y se trasladaban más al interior. ${ }^{7}$ Además, para proporcionar los servicios públicos y privados requeridos por los nuevos pobladores (trabajadores del petróleo y migrantes) comenzaron a surgir nuevas áreas urbanas, que imponían sus propias exigencias a la naturaleza en general (agua, residuos) y a las tierras boscosas en particular: Lago Agrio, Shushufindi, Pompeya, Tarapoa y otras.
El efecto de estas migraciones en el proceso de deforestación ha sido notorio. En 1990 había 13,9 millones de hectáreas de bosques en el país, cifra que descendió a 11,9 millones en el 2000 y a 10,9 en el 2005 (CEPAL, 2007). De acuerdo con las conclusiones del Proyecto PETRAMAZ, en la zona de actividades petrolíferas durante el período 1976-1986 se perdieron más de 26 mil hectáreas de bosque primario, y otras 55.330 en el decenio siguiente, 6.774 de ellas por causas naturales (tornados). Estos datos equivalen a una pérdida media de 3.230 hectáreas en el primer período y 5.532 en el segundo. ${ }^{8}$

La relación entre este proceso de deforestación y la llegada de nuevos colonizadores parece bastante clara: en 1997, el 59,7\% de la pérdida de bosque primario se produjo en una franja de cinco kilómetros en torno a los caminos principales y otro $38,6 \%$ en el kilómetro siguiente. Si se tiene en cuenta el área de influencia de esas actividades, esto equivale a una tasa de deforestación de dos veces a dos veces y media el promedio de deforestación del país, que ya era muy elevado (1,4\% al año en el período 1997 2000 y $1,7 \%$ entre el 2000 y el 2005). Si bien es cierto que este fenómeno se producía principalmente fuera de las áreas protegidas, no solo ponía en peligro las zonas de amortiguación sino en algunos casos las zonas protegidas mismas: en 1993, el gobierno ecuatoriano revocó la designación de área protegida a 50.000 hectáreas de la reserva de Cuyabeno, debido a que los terrenos ya habían sido ocupados por colonos.

\section{IV}

\section{Las consecuencias económicas de la colonización}

Por desgracia, el flujo migratorio no parece haber mejorado gran cosa las condiciones de vida de las personas involucradas. Entre septiembre de 1998 y marzo de 1999 se realizó una encuesta en los dos ejes principales de colonización: las carreteras Chiritza-Tarapoa-Palma Roja (105 km) y Chiritza-Pacayacu-Los Tetetes $(54$ km).

\footnotetext{
${ }^{7}$ El patrón de colonización aplicado por el Instituto Ecuatoriano de Reforma Agraria (IERAC) durante la presidencia de Velasco Ibarra definió la "unidad óptima" como aquella que abarcaba 250 metros a lo largo de la carretera y 2,5 kilómetros de fondo al interior del bosque, con lo cual cada unidad tenía un promedio de 50 hectáreas.
}

En total se encuestaron 1.644 predios $(883+761)$, con una población de 9.248 habitantes (5.211 y 4.037, respectivamente). ${ }^{9}$ De la superficie abarcada por el estudio, 76.600 hectáreas se destinaban a la agricultura o al pastoreo, mientras que 57.000 hectáreas se mantenían en reserva. Alrededor del $45 \%$ de los terrenos cultivables se dedicaban a cultivos permanentes (café, cacao,

\footnotetext{
${ }^{8}$ Véase también Mena, Barbieri y otros (2006).

${ }^{9}$ El estudio fue realizado entre septiembre de 1998 y marzo de 1999 por Maiguashca y Asociados. Cía. Ltda., con arreglo a un contrato suscrito con el proyecto PETRAMAZ.
} 
plátano y banano), el 13\% a cultivos no permanentes (maíz, yuca y arroz) y el $42 \%$ restante al pastoreo. Dadas las características del suelo y el hecho de que se utilizaban técnicas agrícolas que con frecuencia eran inadecuadas (más aptas para las tierras de origen de los inmigrantes), los rendimientos eran muy inferiores al promedio nacional:

- en el caso del café, principal producto comercializado del área, el rendimiento medio solo alcanzaba el 66\% del promedio nacional (que de acuerdo con los estándares internacionales también era bajo).

- en cuanto a la yuca, que ocupaba el segundo lugar en importancia, el rendimiento medio llegaba solo al $62 \%$ del promedio nacional.

- por último, en los productos cultivados para el consumo familiar los rendimientos eran aún más desalentadores: $20 \%$ del promedio nacional en el caso del arroz, $30 \%$ en el del maíz, $40 \%$ en el de los bananos y $30 \%$ en el de los plátanos.

Los beneficios sociales ligados a la generación de empleo eran difíciles de apreciar, dada la muy reducida proporción de personas que trabajaba en actividades relacionadas con el petróleo (menos del 1\% del total de ocupados).

En vista de lo anterior, no sorprende averiguar que según el Banco Interamericano de Desarrollo (BID, 2001) la región amazónica septentrional fuera una de las más deprimidas del país, ya que la indigencia afectaba al $57,3 \%$ de la población y la pobreza al $79 \%$, siendo que el promedio de pobreza en las zonas rurales del país era del $47 \%$. En el mejor de los casos, los servicios de salud eran escasos (Ministerio de Desarrollo Urbano y Vivienda, 1999): la proporción de habitantes de las zonas rurales de la región que tenían acceso a sistemas de agua potable era del $12 \%$ ( $80 \%$ en las zonas urbanas); de las que contaban con sistemas de alcantarillado, un $2 \%$ (28\% en las zonas urbanas), y de las que disponían de servicios de recogida de basuras, un 3\% (59\% en las zonas urbanas). La incidencia de la diarrea en el área se elevaba a 2.453 casos por cada 100.000 habitantes, frente a 1.315 y 1.724 , respectivamente, en las provincias de Pichincha y Guayas (Ministerio de Salud Pública, 2000).

\section{Consecuencias económicas y ambientales de la deforestación}

Por otra parte, los bosques tropicales lluviosos proporcionan un conjunto de servicios que se perderían con la deforestación. Tales servicios cumplen las funciones que se enumeran a continuación.

\section{Funciones económicas}

En esta categoría incluimos los bienes y servicios que son proporcionados por los bosques tropicales y que tienen precios de mercado. Entre ellos cabe mencionar en especial la madera y los productos no maderables (frutas, animales, plantas ornamentales, medicamentos, látex) para la población local. Estos productos se destinan tanto al consumo propio como al pequeño comercio local. Las actividades de extracción de madera y de producción de bienes distintos de la madera, sean ellas sustentables o no (como las mineras), son las de mayor interés.

Grimes, Loomis y otros (1994) realizaron un experimento en tres terrenos de una hectárea de bosque perenne de la estación biológica de Jatum Sacha, ubicada cerca de la reserva de Cuyabeno, en la ribera sur del río Napo. Dos de los terrenos estudiados se hallaban en bosque de tierra firme, con suelos de arcilla roja, a aproximadamente 400 metros sobre el nivel del mar, mientras que el tercero se encontraba en la ribera del río Napo, en bosques aluviales de 350 metros de elevación. Los autores citados calcularon el valor neto obtenido de siete frutos, tres cortezas medicinales y una resina, teniendo presente el precio de estos productos u otros muy similares en el mercado local, su distancia media a los mercados locales (30 kilómetros), el medio de transporte (autobús) y el tiempo requerido para su extracción, transporte y venta. El valor neto anual obtenido de los tres sitios fue de 146,93 dólares, 136,06 dólares y 62,87 dólares, respectivamente. ${ }^{10}$ Por otra parte, el

\footnotetext{
${ }^{10}$ Esta cifra es igual a la sugerida por Torras (2000). Además, se sitúa dentro del rango registrado por van Beukering, Cesar y Janssen (2003): 5 dólares por hectárea en la Amazonia brasileña y 422 dólares en la selva peruana.
} 
valor en madera del terreno más próximo a la carretera era de 163 dólares pero con un ciclo de rotación de 40 años para ser sustentable el valor actualizado neto por hectárea de la madera que contenía sería de solo 189 dólares (aplicando una tasa de descuento del 5\%), cifra muy diferente de la indicada antes para los productos distintos de la madera $(2.939,2.721$ y 1.257 dólares, respectivamente). En consecuencia, las actividades de extracción de madera resultan muy poco rentables si se las lleva a cabo de manera sustentable. ${ }^{11}$

\section{Funciones de atracción turística y esparcimiento}

Los bosques tropicales suelen tener gran atractivo como base del ecoturismo. Si este es el caso, preservarlos puede acarrear dos ventajas importantes a la sociedad. Por una parte, los turistas generalmente obtienen un beneficio neto en concepto de excedente del consumidor: una diferencia positiva entre su disposición a pagar por el disfrute de la experiencia y lo que pagan directa e indirectamente (tiempo) por conseguirla. Por otra, esta nueva actividad económica tiene un efecto multiplicador que favorecerá al país en su conjunto y quizá también a la población local. Las actividades de ecoturismo generan ingresos, crean empleo y producen divisas y el turismo es fuente importante de ellas para la economía del país. Pese a sus restricciones al número de visitantes, las islas Galápagos se sitúan a la vanguardia al respecto. Sin embargo, el segundo destino más popular entre los turistas que visitan el país es justamente la reserva de Cuyabeno. El cuadro 2 muestra el número de turistas que visitaron la zona en el decenio de $1990 .^{12}$

Estos turistas, en su mayoría extranjeros, pagan 20 dólares por entrar en temporada alta (un dólar si son ecuatorianos). La corriente de visitantes es atendida por 18 empresas autorizadas que cobran entre $30 \mathrm{y}$ 120 dólares diarios por una visita de tres a cinco días (un valor medio de 45 dólares diarios) y que en 1998 pagaron 8.800 dólares por operar en la zona. Por último, según Galvin (2000), los turistas extranjeros que visitan la reserva gastan en promedio 369 dólares (una media de 240 dólares).

Varios autores ${ }^{13}$ han efectuado análisis esclarecedores de los diferentes grupos involucrados en la actividad con el fin de determinar de qué manera las variaciones del valor económico total de las áreas naturales afectarán a la población local, a las empresas madereras y a otros. Algunas de las cifras que obtuvieron pueden dar una idea de la importancia de esta clase de turismo para la población local. En 1998, la comunidad siona de Puerto Bolívar (160 personas) obtuvo un total de 104.000 dólares, principalmente por el alquiler de canoas y cabañas. Las comunidades quechuas de Zancudo (110 personas) y de Playas de Cuyabeno (175 personas) ganaron 62.800 y 39.600 dólares, respectivamente, en su mayor parte por pagos

Ecuador: número de turistas que visitaron la reserva de Cuyabeno (Cifras oficiales)

\begin{tabular}{|c|c|c|c|c|}
\hline Año & $\begin{array}{c}\text { Turistas } \\
\text { ecuatorianos }\end{array}$ & Turistas extranjeros & Total & $\begin{array}{c}\text { Porcentaje de } \\
\text { turistas extranjeros }\end{array}$ \\
\hline 1989 & 173 & 581 & 754 & 77 \\
\hline 1990 & 333 & 644 & 977 & 66 \\
\hline 1991 & 612 & 855 & 1467 & 58 \\
\hline 1992 & 945 & 1433 & 2378 & 60 \\
\hline 1993 & 820 & 1484 & 2304 & 64 \\
\hline 1995 & 1582 & 3375 & 4957 & 68 \\
\hline 1996 & 1994 & 5445 & 7439 & 73 \\
\hline 1997 & 1239 & 4410 & 5649 & 78 \\
\hline 1998 & 1696 & 6118 & 7814 & 78 \\
\hline
\end{tabular}

Fuente: Galvin (2000).

\footnotetext{
11 Véase, por ejemplo, Seroa da Motta y Ferraz do Amaral, 2000. Smith, Mourato y otros (2000) también encontraron que en la Amazonia peruana los colonos daban más importancia a la agricultura de corte y quema que a la extracción de madera de forma sustentable (véase también Henrich, 2000).
}

\footnotetext{
12 Es posible que en la práctica la suma sea levemente más alta, ya que un número no registrado de visitantes evita el pago de las tarifas legales.

${ }^{13}$ Por ejemplo, van Beukering, Cesar y otros, 2003.
} 
de los operadores turísticos y venta de artesanía, en tanto que la comunidad cofán, de Zábalo (115 personas) obtuvo ingresos por un total cercano a los 40.000 dólares (Galvin, 2000).

Además, aplicando los métodos de valoración contingente y del costo de viaje, los analistas pueden calcular el beneficio neto en concepto de excedente del consumidor que obtienen los turistas. Puede partirse de la base de que tal beneficio es de 30 dólares por visitante. ${ }^{14}$ Sin embargo, este excedente del consumidor no se perderá, porque los turistas se trasladarán a otro lugar y obtendrán el mismo beneficio. Es difícil predecir si se desplazarán a otro destino dentro del país, pero no puede darse por hecho. En este caso, la pérdida neta solo consistirá en la inversión necesaria en las nuevas instalaciones y los ingresos netos que se pierden hasta que dichas instalaciones comiencen a funcionar. En el presente estudio, para simplificar, se supondrá que los ecoturistas se trasladarán a un lugar alternativo en otro país.

Finalmente, hay un valor intrínseco que las personas atribuirán a la conservación de la reserva. Si la disposición a pagar para mejorar las medidas de conservación pertinentes puede considerarse una variable sustitutiva adecuada de dicho valor, es interesante señalar que Galvin (2000) encontró que, en general, las personas que visitaban la reserva estaban dispuestas a pagar 35 dólares adicionales con ese fin.

\section{Funciones ecológicas}

Los bosques tropicales cumplen numerosas funciones ecológicas. Entre ellas cabe destacar tres. Primero, protegen contra la erosión del suelo por la acción del agua y el viento, que afecta las tierras cultivadas, el costo de mantenimiento de la infraestructura y la vida económica de las presas, además de la calidad del agua. En segundo lugar, son un depósito natural de carbono y por lo general transformarlos en tierras agrícolas y de pastoreo significará una pérdida a este respecto. Tercero, también

\footnotetext{
${ }^{14}$ Baldares, Laarman y Laarman (1990) comprobaron que en Costa Rica los turistas estaban dispuestos a pagar 30 dólares adicionales por visitar áreas naturales protegidas, valor muy semejante a los 35 dólares obtenidos por Tobias y Mendelsohn (1991) respecto de las mismas áreas. Adger, Brown y otros (1995) dan una cifra de 3 dólares por visita en el caso de los turistas que visitaron bosques nativos de México dentro de un paquete que incluía otros rubros, y 70 dólares cuando solo les interesaban los bosques.
}

son un depósito de diversidad biológica. Por ejemplo, la reserva de Cuyabeno contiene una de las mayores diversidades de árboles por hectárea (473 especies), además de 514 especies de aves, 117 de mamíferos y 176 de reptiles.

En realidad, resulta difícil medir la pérdida de diversidad biológica y aún más apreciarla en términos económicos. Desde el punto de vista de su papel en el desarrollo de medicamentos y de productos farmacéuticos nuevos, y aplicando en líneas generales la metodología propuesta por Pearce y Puroshothaman (1992), la literatura especializada ha propuesto una amplia gama de valores que van desde 7 dólares por hectárea (Ruitenbeck, 1992) a 20 dólares (Fearnside, 1997; Mendelsohn, 1994). Por su parte, Adger, Brown y otros (1995) mencionan una cifra similar a la primera (6,4 dólares por hectárea) pero dentro de una gama de valores mucho más amplia, que fluctúa entre 1 y 90 dólares.

En cuanto a la pérdida de depósitos de carbono que podría acarrear la deforestación, si se asigna un valor de 10 dólares a la tonelada de carbono, transformar una hectárea de bosque abierto en terreno de cultivo o de pastoreo cuesta entre 300 y 500 dólares (Brown, Pearce y otros, 1993). El costo de transformar un bosque cerrado secundario en tierras agrícolas o de pastoreo costará de 1.000 a 1.500 dólares por hectárea y, finalmente, si lo que se transforma es un bosque tropical primario, la pérdida ascenderá a 2.000 dólares por hectárea.

\section{Funciones culturales}

Por último, hay que recordar que algunos relacionan los bosques tropicales con su propia identidad cultural. Así, cuando tales bosques desaparecen no solo se pierden conocimientos tradicionales, a menudo muy apreciados por los ancianos de las comunidades locales, sino identidad y diversidad culturales.

La transformación de los bosques tropicales debido a la extracción de petróleo y actividades conexas, tanto directas como inducidas, provocará la pérdida total o parcial de la capacidad del ecosistema de seguir cumpliendo estas funciones. ${ }^{15}$

\footnotetext{
15 Estas cifras coinciden con las obtenidas por Adger, Brown y otros (1995) y Pearce y Moran (1994), esto es, de 650 a 3.400 dólares por hectárea. Cabe tener en cuenta que estos autores utilizan un valor diferente (20 dólares) por tonelada de carbono.
} 


\section{VI}

\section{El costo económico de explotar yacimientos nuevos: una simulación}

Como ya se señaló, en la región oriental la deforestación se ha producido principalmente fuera de la zona protegida, aunque ya está acercándose a sus límites.

Sin embargo, el futuro se presenta mucho más sombrío. Se están otorgando licencias para explorar y explotar nuevos campos petrolíferos al oriente de los existentes, incluso al interior de "zonas intangibles" (que son objeto de máxima protección). Entre esos campos destaca el de TIT (Tiputuni-Ipishingo-Tambococha). Es posible que contenga 920 millones de barriles de baja calidad y alta densidad —es decir, un $20 \%$ de las reservas de petróleo del país y que pueda producir 100 mil barriles diarios durante 12 años y después una cantidad decreciente durante otros 13 años, con un costo de 12 dólares por barril. Es innegable que Ecuador tiene derecho a explotar sus recursos naturales y obtener así las divisas que tanto necesita; sin embargo, conviene analizar cuál es la mejor manera de proceder cuando se enfrenta un dilema como este. La experiencia del pasado podría ayudarnos.

Para ello, podría ser de utilidad formarse una idea del valor económico que se perdería si se produce deforestación en las reservas de Cuyabeno y Yasuni.

Como se dijo al comienzo del presente estudio, las cosas pueden hacerse de otra manera: es posible extraer petróleo tratando de evitar la deforestación. Sin duda que esto resultaría más caro pero ¿no sería razonable hacerlo si se piensa en el daño ambiental evitado? ¿Qué inversión se justificaría económicamente para evitar esta pérdida para el medio ambiente? Como ya se señaló, no se pretende dar aquí respuestas categóricas, sino simplemente mostrar que tal vez valga la pena plantearse estas preguntas. Una simulación sencilla podría llevarnos a una primera y burda aproximación.

Para ello, supondremos que abrir estos nuevos yacimientos implicará tener que construir caminos para conectarlos con los principales centros de actividad. En este caso, cuando entren en funcionamiento los nuevos campos petrolíferos, en la zona se producirá una deforestación similar a la experimentada en el pasado. Ahora bien, supóngase que se aplican los valores citados a una zona que, debido a las actividades relacionadas con la explotación de petróleo (incluidas la migración y la urbanización), tiene una tasa de deforestación del $8 \%$ al año (para simplificar la supondremos constante) con un valor inicial de 2 mil hectáreas taladas el primer año. Este valor es, en promedio, similar al registrado en torno a los yacimientos originales (Mena, Barbieri y otros, 2006, cuadro 3).

A continuación, para completar el cálculo habría que introducir el valor individual de cada uno de los servicios. De todas las funciones económicas mencionadas que cumplen los bosques tropicales solo se estimará el valor de cuatro: la producción de madera y de productos distintos de esta, el ecoturismo, la preservación de la diversidad biológica y la fijación de carbono. No se tendrán en cuenta la protección contra las crecidas, la erosión y los incendios, ni la producción de agua. En consecuencia, el valor resultante subestimará claramente los costos, pero servirá para calcular el monto de la inversión que se justificaría, en términos de eficiencia, para tratar de minimizarlos.

Lo ideal sería relacionar la pérdida de cada una de estas funciones con una medida cuantitativa de deforestación: por ejemplo, por hectárea u otra medida de superficie. Esto será posible casi únicamente respecto de funciones tales como la absorción de carbono, porque las funciones dosis-respuesta son no lineales y generalmente abarcan una gama crítica. No tiene sentido tratar de estimar el valor económico de la biodiversidad por hectárea, porque depende de qué hectárea se trate. No hay duda de que la primera tendrá un valor de cero, mientras que la que se aproxime al umbral crítico tendrá el valor máximo asignado a este tipo de diversidad biológica. Entre ellas hay una función cuya forma simplemente ignoramos, pese a lo cual supondremos un valor dado por hectárea. ${ }^{16}$ Por otra parte, se puede determinar la cantidad de madera y productos maderables que contiene en promedio una hectárea de bosque tropical pero ello no quiere decir que si esta hectárea se convirtiera en tierra cultivable

\footnotetext{
16 Para simplificar, van Beukering, Cesar y otros (2003) también partieron del supuesto de que las funciones dosis-efecto (funciones de respuesta del ecosistema) son lineales, no muestran irreversibilidad y no se ven afectadas por umbrales.
} 
la población local perdería esos productos. Con demasiada frecuencia el costo real será simplemente el costo de oportunidad del tiempo adicional necesario para obtener estos productos desde una distancia un poco mayor. También en este caso supondremos que el producto se pierde o, alternativamente, que el valor marginal del tiempo es igual al valor del producto que dejó de obtenerse.

En consecuencia, se utilizarán los siguientes valores unitarios:

- Productos distintos de la madera: 115,3 dólares por hectárea (promedio del ingreso obtenido en los tres terrenos de la zona que fueron estudiados).

- Ecoturismo: una pérdida de 20 dólares por visitante (suma pagada en dólares por los turistas extranjeros tanto al gobierno de Ecuador como a la población local). Suponemos un número inicial de 5.000 visitantes a la zona, que aumentan a razón del 5\% al año y que no sobrepasa una capacidad de absorción de 20.000 visitantes (el 80\% de ellos extranjeros).

- Biodiversidad: 7 dólares por hectárea (valor correspondiente al rango inferior de los citados en la literatura especializada).
- Retención de carbono: pérdida de 200 toneladas métricas de carbono por cada hectárea de bosque tropical convertido en tierras cultivables, a razón de 25 dólares por tonelada.

En el supuesto de que la tasa de actualización social sea del $4 \%$, el valor actualizado neto (VAN) del costo ambiental asociado al proceso de deforestación sería el siguiente:

$$
\text { VAN }=451 \text { millones de dólares }
$$

Por cierto, el cuadro 3 simplemente ilustra el hecho de que desde el punto de vista social se justifica realizar una inversión para tratar de mitigar este costo. Por una parte, es sencillo realizar un análisis de sensibilidad que ayude a identificar algunos valores críticos. Por ejemplo, si asignamos un valor de 10 dólares por hectárea a los productos distintos de la madera, porque consideramos que 115,3 es demasiado alto, el VAN solo bajará a 421 millones de dólares. Por otra parte, si tomamos el valor de cada tonelada de carbono, que es variable, el cambio será mucho más evidente.

Sin embargo, hay otros dos aspectos que vale la pena considerar, lo que se hará en las dos secciones siguientes.

Ecuador: valor económico de la deforestación y componentes principales ${ }^{a}$ (Dólares)

\begin{tabular}{|c|c|c|c|c|c|c|c|c|}
\hline & $\begin{array}{c}\text { Área } \\
\text { deforestada } \\
\text { (hectáreas) }\end{array}$ & $\begin{array}{c}\text { Valor de los } \\
\text { productos distintos } \\
\text { de la madera (115,3 } \\
\text { dólares por ha) }\end{array}$ & $\begin{array}{l}\text { Número de } \\
\text { visitantes }\end{array}$ & $\begin{array}{l}\text { Valor del } \\
\text { ecoturismo } \\
\text { (20 dólares } \\
\text { por visitante) }\end{array}$ & $\begin{array}{c}\text { Carbono } \\
\text { liberado } \\
\text { (en toneladas) }\end{array}$ & $\begin{array}{c}\text { Valor del carbono } \\
\text { liberado ( } 25 \text { dólares } \\
\text { la tonelada de } \mathrm{CO}_{2} \\
\text { equivalente) }\end{array}$ & $\begin{array}{c}\text { Valor de la } \\
\text { biodiversidad } \\
\text { perdida } \\
\text { (7 dólares por ha) }\end{array}$ & $\begin{array}{l}\text { Costo total } \\
\text { (en dólares) }\end{array}$ \\
\hline 1 & 2000 & 230600 & 6000 & 120000 & 400000 & 7334000 & 14000 & 7698600 \\
\hline 5 & 2431 & 280296 & 7293 & 145861 & 486203 & 8914523 & 17017 & 9357696 \\
\hline 10 & 3103 & 357736 & 9308 & 186159 & 620531 & 11377441 & 21719 & 11943055 \\
\hline 15 & 3960 & 456572 & 11880 & 237592 & 791973 & 14520818 & 27719 & 15242701 \\
\hline 20 & 5054 & 582715 & 15162 & 303234 & 1010780 & 18532653 & 35377 & 19453979 \\
\hline 25 & 6450 & 743708 & 19351 & 387012 & 1290040 & 23652883 & 45151 & 24828754 \\
\hline 30 & 8232 & 949181 & 20000 & 400000 & 1646454 & 30187738 & 57626 & 31594545 \\
\hline 35 & 10507 & 1211422 & 20000 & 400000 & 2101339 & 38528054 & 73547 & 40213023 \\
\hline 40 & 13410 & 1546116 & 20000 & 400000 & 2681900 & 49172645 & 93867 & 51212627 \\
\hline 45 & 17114 & 1973279 & 20000 & 400000 & 3422860 & 62758140 & 119800 & 65251219 \\
\hline 50 & 21843 & 2518459 & 20000 & 400000 & 4368533 & 80097057 & 152899 & 83168415 \\
\hline
\end{tabular}

Fuente: elaboración propia.

a El cuadro presenta los principales resultados de estos supuestos para un período de 50 años. 


\section{VII}

\section{El valor de la inversión en un país en desarrollo}

El ejercicio anterior sería razonablemente aceptable en el caso de economías desarrolladas que se encuentran en situación de equilibrio intertemporal y con una tasa óptima de ahorro. En cambio, no lo es en el caso de los países en desarrollo, donde los beneficios invertibles tienen una prima sobre aquellos destinados al consumo: es decir, la productividad social marginal del capital (la tasa de interés contable, que indica la variación en el tiempo del valor social de la inversión) es superior a la tasa de interés del consumo. En otras palabras, la productividad social marginal del capital $p$ es superior a la tasa de actualización del consumo $i$ (Azqueta, 2007, cap. 5).

Si esto es así, los efectos ambientales que dan lugar al aumento (o disminución) de las posibilidades de consumo presente de una sociedad en su conjunto no deben tratarse de la misma manera que aquellos que influyen en las corrientes de inversión (consumo futuro). ${ }^{17}$

Una manera simple de tener en cuenta este hecho es separar claramente todos los rubros que corresponden a costos y beneficios entre los que influyen en las posibilidades de consumo presente y los que modifican los recursos para inversión, para luego introducir una prima sobre esas corrientes de inversión y finalmente sumarla a los más convencionales beneficios netos del consumo. Hecho esto, la tasa de actualización del consumo se utilizaría para calcular el valor actualizado neto de las consecuencias ambientales en su conjunto. Esta prima $(\lambda)$ toma aproximadamente la forma de la siguiente ecuación (Azqueta, 1985, pp. 97-101):

$$
\lambda=\frac{\left(1-s^{r}\right) \rho}{i-s^{r} \rho}
$$

siendo $s^{r}$ la tasa de reinversión de los beneficios de la inversión.

Tradicionalmente, el valor de la productividad social marginal del capital $(p)$ se ha obtenido ya sea analizando la tasa rentabilidad interna de los mejores proyectos de inversión pública que han de ser rechazados por falta de

\footnotetext{
${ }^{17}$ Naturalmente, la misma salvedad se aplica a las exportaciones de petróleo (directa o indirectamente producen fondos para inversión), pero como no estamos proponiendo detenerlas sino realizarlas de distinta manera, no es necesario ahondar en este punto.
}

recursos o bien, desde el punto de vista macroeconómico, examinando la sobrevaluación de la moneda nacional: el precio de cuenta de las divisas. Ahora que muchos países en desarrollo han liberalizado sus mercados de divisas y sus corrientes de importaciones, la situación es algo diferente:

i) en los países que han liberalizado los mercados de divisas pero aún no han hecho lo mismo con los flujos comerciales, el precio de cuenta de las divisas (que refleja el mayor precio interno en relación con su precio internacional en un conjunto representativo de productos básicos) sigue representando esta sobrevaluación oculta de la moneda local.

ii) algunos países han desregulado tanto las importaciones como los mercados de divisas. Esto no quiere decir, sin embargo, que su tasa de ahorro sea óptima. Es probable que el gobierno tenga serias limitaciones presupuestarias y al mismo tiempo enfrente una crisis de la deuda externa y vaya postergando las inversiones de valor social. Teniendo presente la relación entre ambos déficit (interno y externo), una buena indicación del valor social de las divisas sería el precio que tienen que pagar las autoridades por el acceso a crédito en los mercados financieros internacionales: el diferencial de la tasa de interés que cobrarían los mercados interno y externo.

En consecuencia, hay maneras de indicar de forma cuantitativa el hecho de que en las economías en desarrollo las posibilidades de inversión (principalmente en términos de divisas) tienen una prima. Aplicando un criterio conservador, partiremos de la base de que $\lambda=2$, es decir, que la productividad social marginal del capital es igual al doble de la tasa social de descuento, que es del 4\%; pero no habría problema en modificar este valor como parte de un análisis de sensibilidad. Por lo tanto, una vez que se han determinado las consecuencias ambientales y se les ha asignado un valor adecuado, hay que clasificarlas en las dos categorías mencionadas: las que se relacionan con el consumo presente y las que afectan las posibilidades de inversión. Para obtener el valor neto actualizado de las consecuencias ambientales hay que multiplicarlas por el correspondiente precio de cuenta de la inversión y actualizar el resultado. 
La producción de artículos distintos de la madera para el consumo propio o para su comercialización en los mercados locales puede fácilmente considerarse como un beneficio del consumo. Por otra parte, mientras el país pueda cobrar a alguien este servicio, puede considerarse que tanto la conservación de la biodiversidad como la retención de carbono son beneficios de la inversión. Sería el caso, por ejemplo, de una empresa farmacéutica que pague por asegurar su acceso al territorio, o que en el marco del Protocolo de Kyoto de la Convención Marco de las Naciones Unidas sobre el Cambio Climático se reconozca el papel que desempeñan los bosques. Lo mismo puede aplicarse a los dólares que pagan los turistas extranjeros por ingresar a la zona: alivian los problemas de balanza de pagos.

Teniendo presente lo anterior y suponiendo, contra todas las indicaciones, que Ecuador podrá alcanzar la tasa óptima de ahorro en 50 años, de tal manera que una vez más $\lambda$ sea igual a 1 , el valor actual del costo ambiental asociado a la deforestación será

$$
\text { VAN }=624 \text { millones de dólares }
$$

esto es, tendrá un incremento del $40 \%$.

\section{VIII}

\section{Actualización de las consecuencias ambientales}

Descontar consecuencias ambientales que podrían durar mucho tiempo e incluso ser irreversibles no es fácil. En la práctica, ello tiene que ver con un problema de equidad intergeneracional en condiciones de incertidumbre, unido al hecho de que el valor presente de las consecuencias que surgen a muy largo plazo es muy próximo a cero. La manera tradicional de calcular la tasa social de descuento (la tasa de actualización del consumo: $i$ ) se basaba a la vez en la tasa prevista de crecimiento del ingreso $g$ y la utilidad marginal del consumo global $\eta$, a lo que a veces se sumaba un factor puro de preferencia por el tiempo $\delta$ :

$i=g \cdot \eta+\delta$
en que $\eta=\frac{C}{d U / d C} \frac{d(d U / d C)}{d C}$

siendo $C$ el nivel de consumo y $U$ el nivel de utilidad social.

Sin embargo, a muy largo plazo, utilizar un factor convencional de descuento basado en esta tasa (por ejemplo, $e^{-i t}$ ) significaría que el bienestar de las generaciones futuras carece de importancia para la decisión que hay que adoptar ahora. Por otra parte, utilizar una tasa de descuento de cero equivaldría a sostener que sea cual fuere la mejor situación en que se encuentren las generaciones futuras en cuanto a la satisfacción de sus necesidades, toda mejora que reciban tendría el mismo valor en términos del bienestar social actual que si debiera percibirlo la generación actual (algo que nos inclinamos a rechazar por motivos de equidad). En consecuencia, para evitar no solo la tiranía del presente (tasas de descuento positivas) sino también la del futuro (una tasa de descuento igual a cero) se ha recomendado aplicar factores de descuento hiperbólicos (Chichilnisky, 1996; Heal, 1998):

$$
e^{-i \log t}
$$

Este procedimiento, que no varía el valor de la tasa social de descuento, tiene la ventaja de que el factor de actualización comienza con un valor positivo pero luego tiende asintóticamente a cero. Además, parece concordar con la opinión de los especialistas (Weitzman, 2001).

Por lo tanto, si introducimos en el proceso un factor de descuento hiperbólico, en la forma sencilla sugerida por Weitzman (2001), el valor presente de la pérdida será

$$
\text { VAN }=1.265 \text { millones de dólares }
$$

Finalmente, si se aplican simultáneamente ambos elementos (prima de la inversión y factor de actualización hiperbólico), tendremos que

$$
\text { VAN }=1.601 \text { millones de dólares }
$$

En el cuadro 4 se resumen los principales resultados de este procedimiento, partiendo de supuestos diferentes. Como puede verse, el resultado final es muy sensible al valor del precio de una tonelada de $\mathrm{CO}_{2}$ equivalente, $\mathrm{y}$ a la tasa de actualización, mientras que, por ejemplo, el valor de los productos distintos de la madera no influye demasiado en el costo final de la deforestación. La 
CUADRO 4

Ecuador: valor total de la deforestación ${ }^{a}$

(Millones de dólares)

ANÁLISIS DE SENSIBILIDAD: PRINCIPALES RESULTADOS

\begin{tabular}{|c|c|c|c|c|c|}
\hline & & $\lambda=1$ & $\lambda=1$ & $\lambda>1$ & $\lambda>1$ \\
\hline & & $\begin{array}{c}\text { Valor del } \mathrm{CO}_{2} \\
\text { equivalente: } \\
25 \text { dólares la tonelada }\end{array}$ & $\begin{array}{c}\text { Valor del } \mathrm{CO}_{2} \\
\text { equivalente: } \\
35 \text { dólares la tonelada }\end{array}$ & $\begin{array}{c}\text { Valor del } \mathrm{CO}^{2} \\
\text { equivalente: } \\
25 \text { dólares la tonelada }\end{array}$ & $\begin{array}{c}\text { Valor del CO2 } \\
\text { equivalente: } \\
35 \text { dólares la tonelada }\end{array}$ \\
\hline$r=4 \%$ & $\begin{array}{l}\text { Valor de productos } \\
\text { distintos de la madera: } \\
10 \text { dólares por ha }\end{array}$ & 421 & 586 & 595 & 828 \\
\hline$r=4 \%$ & $\begin{array}{l}\text { Valor de productos } \\
\text { distintos de la madera: } \\
115,3 \text { dólares por ha }\end{array}$ & 451 & 616 & 624 & 857 \\
\hline $\begin{array}{l}r=4 \%(\text { años } 1-5) \\
r=3 \%(\text { años } 6-25) \\
r=2 \%(\text { años } 26-50)^{b}\end{array}$ & $\begin{array}{l}\text { Valor de productos } \\
\text { distintos de la madera: } \\
10 \text { dólares por ha }\end{array}$ & 1178 & 1641 & 1514 & 2110 \\
\hline $\begin{array}{l}r=4 \%(\text { años } 1-5) \\
r=3 \%(\text { años } 6-25) \\
r=2 \%(\text { años } 26-50)\end{array}$ & $\begin{array}{l}\text { Valor de productos } \\
\text { distintos de la madera: } \\
115,3 \text { dólares por ha }\end{array}$ & 1265 & 1728 & 1601 & 2197 \\
\hline
\end{tabular}

Fuente: elaboración propia.

a $\mathrm{r}=$ tasa de descuento.

b Recomendado en Weitzman (2001)

prima sobre la inversión se sitúa en algún punto entre ambos e influye moderadamente en el resultado final.

Los valores fluctúan entre 421 millones y 2.197 millones de dólares. Estas cifras pueden compararse tanto con el valor actualizado neto de las reservas

\section{IX}

Conclusiones

Las exportaciones de petróleo proporcionan las divisas tan necesarias para algunos países en desarrollo altamente endeudados. Sin embargo, la extracción de petróleo puede acarrear graves consecuencias ambientales. Ecuador es un buen ejemplo al respecto: la explotación del petróleo de la región amazónica ha estado acompañada de un acentuado proceso de deforestación. Ahora que los yacimientos se aproximan al término de su vida económica, el país está otorgando licencias para explorar y explotar otros nuevos, próximos a zonas de gran valor ecológico. Teniendo en cuenta que la mayor parte del proceso de deforestación se ha debido a la llegada de colonos por los caminos que se han construido en relación con las instalaciones petrolíferas, cabe plantearse la siguiente cuestión: ¿se justificaría desde el punto de vista eco- existentes del campo petrolífero de TIT, esto es, 3.500 millones de dólares, como con la cifra solicitada por el presidente Correa de Ecuador, el 5 de junio de 2007, para no explotar este yacimiento: 1.750 millones de dólares. ${ }^{18}$ nómico buscar nuevas maneras de extraer petróleo sin construir carreteras?

Para allanar el camino hacia una posible respuesta a esta pregunta se ha realizado una simulación en que se introducen algunos valores económicos asociados a la pérdida ambiental provocada por la deforestación. Se demuestra que las pérdidas directas e indirectas vinculadas a la deforestación, debidas principalmente a las corrientes migratorias inducidas hacia el área,

\footnotetext{
18 Paula Suárez, asesora del Ministerio de Energía y Minas de Ecuador, trabajo presentado al seminario sobre energía y áreas protegidas, organizado por la CEPAL y The Nature Conservancy, Santiago de Chile, junio de 2007.
} 
posiblemente sean cuantiosas. Los países en desarrollo difícilmente pueden prescindir de los ingresos que les proporcionan las exportaciones de petróleo, particularmente si se considera que el mundo desarrollado es muy renuente a sustituirlas por pagos relacionados con los servicios ambientales. En consecuencia, lo más probable es que en el futuro estas exportaciones sigan cumpliendo una función vital.

Sin embargo, dicho esto, lo que revelan los costos ambientales relacionados con las exportaciones de petróleo es el beneficio social que puede lograrse (así como el monto de la inversión que se justifica y la diferencia de costos en que se incurre) si se minimizan estas consecuencias. Por ejemplo, si el país está considerando la posibilidad de permitir que las empresas petrolíferas exploren nuevos terrenos porque los ya explotados comienzan a dar señales de agotamiento, habría que sopesar las ventajas de invertir en explotar más a fondo (y a mayor costo) las reservas recuperables de los pozos ya abiertos y solo entonces poner en explotación terrenos nuevos, pero sin construir nuevos caminos (en este caso, también con un mayor costo de inversión).

\section{Bibliografía}

Adger, W.N., K. Brown y otros (1995): Total economic value of forest in Mexico, Ambio, vol. 24, $\mathrm{N}^{\circ}$ 5, Estocolmo, Royal Swedish Academy of Sciences.

Agencia Internacional de Energía (varios años): Oil Market Report, París.

Azqueta, D. (1985): Teoría de los precios sociales, Madrid, Instituto Nacional de Administración Pública.

(2007): Introducción a la economía ambiental, Madrid, McGraw-Hill.

Baldares, C., M.J. Laarman y J.G. Laarman (1990): User fees at protected areas in Costa Rica, en J.R. Vincent, E.W. Crawford y J.P. Hoehn (comps.), Valuing Environmental Benefits in Developing Economies, Ann Arbor, Michigan, Michigan State University.

BID (Banco Interamericano de Desarrollo) (2001): Ecuador: Documento de país, Departamento Regional de Operaciones 3, diciembre de 2001, Washington, D.C.

Brown, K., D.W. Pearce y otros (1993): Economics and the Conservation of Global Biological Diversity, Working Paper, $\mathrm{N}^{\circ} 2$, Washington, D.C., Global Environment Facility.

Brown, L.A., S. Digiacinto y otros (1996): Urban system development, Ecuador's Amazon Region, and generalization, en Y. Gradus y H. Lithwick (comps.), Frontiers in Regional Development, Lanham, Maryland, Rowman and Littlefield Publishers Inc.

CEPAL (Comisión Económica para América Latina y el Caribe) (2006): Panorama de la inserción internacional de América Latina y el Caribe, 2005-2006, LC/G.2313-P, Santiago de Chile. Publicación de las Naciones Unidas, $\mathrm{N}^{\circ}$ de venta: S.06.II.G.67.

(2007): Anuario estadístico de América Latina y el Caribe, 2006, LC/G.2332-P, Santiago de Chile. Publicación de las Naciones Unidas, $N^{\circ}$ de venta: E/S.07.II.G.1.

Colburn, F.D. (1997): Shrimp or bananas, Journal of Business Research, vol. $38, \mathrm{~N}^{\circ} 1$, Amsterdam, Elsevier.

Costanza, R., R. D'Arge y otros (1997): The value of the world's ecosystem services and natural capital, Nature, vol. 387, Nueva York, Nature Publishing Group.

Chichilnisky, G. (1996): An axiomatic approach to sustainable development, Social Choice and Welfare, vol. 13, № 2, Nueva York, Springer.

Fearnside, P.M. (1997): Environmental services as a strategy for sustainable development in rural Amazonia, Ecological Economics, vol. 20, $\mathrm{N}^{\mathrm{o}} 1$, Amsterdam, Elsevier.

Galvin, T.E. (2000): Monetary Valuation of Nature Tourism in the Cuyabeno Wildlife Reserve, Amazon, Ecuador, tesis de grado, Gainsville, Universidad de Florida.
García, M.F. (2000): Valoración económica de la descongestión turística en los sitios de visita sobreutilizados de las Islas Galápagos, tesis de grado, Quito, Departamento de Economía, Pontificia Universidad Católica del Ecuador.

Goodland, R. (2002): Ecuador: Oleoducto de Crudos Pesados (OCP) (Heavy Crude Oil Pipeline). Independent Compliance Assessment of OCP with the World Bank's Environmental and Social Policies, septiembre.

Grimes, A., S. Loomis y otros (1994): Valuing the rain forest: the economic value of non-timber forest products in Ecuador, Ambio, vol. 23, $\mathrm{N}^{\mathrm{o}}$ 7, Estocolmo, Royal Swedish Academy of Sciences.

Gupta, A. y M.G. Asher (1998): Environment and the Developing World, Chichester, John Wiley.

Heal, G. (1998): Economic Theory and Sustainability, Nueva York, Columbia University Press.

Henrich, J. (2000): Does culture matter in economic behaviour? Ultimatum game bargaining among the Machiguenga of the Peruvian Amazon, American Economic Review, vol. 90, $\mathrm{N}^{\circ} 4$, Nashville, Tennessee, American Economic Association.

Jacôme, L.I. (2004): The Late 1990s Financial Crisis in Ecuador: Institutional Weaknessses, Fiscal Rigidities, and Financial Dollarization at Work, IMF Working Paper, No 04/12, Washington, D.C., Fondo Monetario Internacional.

Londero, E. (comp.) (1992): Precios de cuenta: principios, metodología y estudios de caso, Washington, D.C., Banco Interamericano de Desarrollo.

Mena, C.J., A.F. Barbieri y otros (2006): Pressure on the Cuyabeno Wildlife Reserve: development and land use/cover change in the Northern Ecuadorian Amazon, World Development, vol. 34, $\mathrm{N}^{\circ} 10$, Amsterdam, Elsevier.

Mendelsohn, R. (1994): Property rights and deforestation, Oxford Economic Papers, vol. 46, Oxford, Reino Unido, Oxford University Press.

Ministerio de Desarrollo Urbano y Vivienda. (MIDUVI) (1999): Evaluación nacional de los servicios de agua, saneamiento y alcantarillado, Quito, Subsecretaría de Saneamiento Ambiental.

Ministerio de Salud Pública (2000): Directorio nacional de epidemiología. Informe 1990-2000, Quito.

Pearce, D. y E. Moran (1994): The Economic Value of Biodiversity, Londres, Earthscan.

Pearce, D. y S. Puroshothaman (1992): Protecting Biological Diversity: The Economic Value of Pharmaceutical Plants, Global Environmental Change Working Paper, No 92-27, Londres, Centre for Social and Economic Research on the Global Environment (CSERGE). 
Ruitenbeck, J. (1992): The rainforest supply price: a tool for evaluating rainforest conservation expenditures, Ecological Economics, vol. 6, Amsterdam, Elsevier.

Seroa da Motta, R. y C.A. Ferraz do Amaral (2000): Estimating timber depreciation in the Brazilian Amazon, Environment and Development Economics, vol. 5, Cambridge, Cambridge University Press.

Smith, J., S. Mourato y otros (2000): Willingness to Pay for Environmental Services among Slash and Burn Farmers in the Peruvian Amazon: Implications for Deforestation and Global Environmental Markets, Londres, Centre for Social and Economic Research on the Global Environment (CSERGE).
Tobias, D. y R.O. Mendelsohn (1991): Valuing ecotourism in a tropical rainforest reserve, Ambio, vol. 20, No 2, Estocolmo, Royal Swedish Academy of Sciences.

Torras, M. (2000): The total economic value of Amazonian deforestation, 1978-1993, Ecological Economics, vol. 33, Amsterdam, Elsevier.

Van Beukering, P.J.H., H.S.J. Cesar y M.A. Janssen (2003): Economic valuation of the Leuser National Park on Sumatra, Indonesia, Ecological Economics, vol. 44, Amsterdam, Elsevier.

Weitzman, M.L. (2001): Gamma discounting, American Economic Review, vol. 91, No 1, Nashville, Tennessee, American Economic Association. 